\title{
Hypercholesterolemia and chronic ischemia alter myocardial responses to selective cyclooxygenase- 2 inhibition
}

\author{
Louis M. Chu, MD, ${ }^{\mathrm{a}, \mathrm{b}}$ Michael P. Robich, MD, ${ }^{\mathrm{a}, \mathrm{b}}$ Antonio Lassaletta, MD, ${ }^{\mathrm{a}, \mathrm{b}}$ Thomas Burgess, MS, \\ Yuhong Liu, MD, ${ }^{\mathrm{a}, \mathrm{b}}$ Nicholas Sellke, ${ }^{\mathrm{a}}$ and Frank W. Sellke, $\mathrm{MD}^{\mathrm{a}, \mathrm{b}}$
}

\begin{abstract}
Objective: Cyclooxygenase- 2 inhibitors have been implicated in adverse cardiac events. We hypothesize that hypercholesterolemia and ischemia may alter the myocardial response to the cyclooxygenase- 2 inhibitor celecoxib.
\end{abstract}

\begin{abstract}
Methods: Yorkshire swine fed normal chow $(\mathrm{CX}, \mathrm{n}=6)$ or high-cholesterol diet $(\mathrm{HCX}, \mathrm{n}=6)$ underwent placement of an Ameroid constrictor on the left circumflex artery and were started on celecoxib ( $200 \mathrm{mg} / \mathrm{day})$. After 7 weeks, ischemic and nonischemic myocardium was analyzed for thrombogenic ratio (thromboxane content divided by prostacyclin content), total protein oxidative stress, and expression of prostacyclin synthase, thromboxane synthase, myeloperoxidase, and superoxide dismutase. Cardiac function, tissue perfusion, and vessel density were measured.
\end{abstract}
Results: HCX animals were significantly hypercholesterolemic compared with CX animals. Thrombogenic ra- tio was significantly higher in the HCX group than in the CX group, but prostacyclin and thromboxane synthase expression was similar in all tissues. Myocardial perfusion was decreased in the HCX group compared with the CX group. Total oxidative stress, myeloperoxidase, and superoxide dismutase were increased in ischemic tissue compared with nonischemic tissues, but there was no diet-induced difference between groups. There was no dif- ference in capillary or arteriolar density between groups. Left ventricular contractility was greater in the HCX group than in the CX group, but there was no significant difference in heart rate, mean arterial pressure, or left ventricular pressure.

Conclusions: Hypercholesterolemic patients using celecoxib may be at higher risk for thrombotic events than those with normal cholesterol, but the relationship between dyslipidemia, ischemia, and cyclooxygenase-2 inhibition is likely much more complicated than originally thought. (J Thorac Cardiovasc Surg 2011;142:675-81)

Nonsteroidal anti-inflammatory drugs inhibit the conversion of arachidonic acid into prostaglandin $\mathrm{H}_{2}$ by cyclooxygenase (COX), decreasing downstream synthesis of prostaglandins, which are responsible for the symptoms of pain, inflammation, and fever. COX also converts arachidonic acid into prostacyclin, a vasodilator and inhibitor of platelet aggregation, and thromboxane, a vasoconstrictor and promoter of platelet aggregation. However, COX also helps

From the Division of Cardiothoracic Surgery, ${ }^{a}$ Department of Surgery, Warren Alpert Medical School of Brown University, Providence, RI; and the Division of Cardiothoracic Surgery, ${ }^{\mathrm{b}}$ Department of Surgery, Beth Israel Deaconess Medical Center, Boston, Mass.

Funded by grants from the National Heart, Lung, and Blood Institute (RO1HL46716, RO1HL69024, and RO1HL85647, to Dr Sellke), and National Institutes of Health training grants T32-HL094300 (to Dr Chu), T32-HL007734 and T32-HL76130 (to Dr Robich), and T32-HL076134 (to Dr Lassaletta).

Disclosures: Authors have nothing to disclose with regard to commercial support.

Read at the 91st Annual Meeting of The American Association for Thoracic Surgery, Philadelphia, Pennsylvania, May 7-11, 2011.

Received for publication April 14, 2011; revisions received May 23, 2011; accepted for publication June 6, 2011; available ahead of print July 15, 2011.

Address for reprints: Frank W. Sellke, MD, Division of Cardiothoracic Surgery, Alpert Medical School, Brown University, 593 Eddy St, APC 424, Providence, RI 02903 (E-mail: fsellke@ lifespan.org).

$0022-5223 / \$ 36.00$

Copyright (C) 2011 by The American Association for Thoracic Surgery

doi:10.1016/j.jtcvs.2011.06.005 maintain gastric mucosa and decreases gastric acid production. Thus, the use of traditional COX inhibitors has been limited by increased risk of gastrointestinal complications.

In 1991, COX-2 was discovered, which was found to be induced only at sites of inflammation. ${ }^{1}$ Subsequently, COX2-specific inhibitors were developed to target the undesirable effects of prostaglandins while sparing the protective physiologic effects. Unfortunately, although they did decrease the incidence of gastrointestinal complications, several of these medications were found to increase cardiovascular risk compared with placebo and nonselective COX inhibitors, leading to their withdrawal from worldwide markets. ${ }^{2}$ The majority of these studies, however, did not assess the effect of comorbidities such as hypercholesterolemia or chronic ischemia on the safety profile of COX-2 inhibitors. The COX-2 inhibitors parecoxib and valdecoxib have been found to increase cardiovascular and other major complications compared with placebo after coronary artery bypass surgery ${ }^{3,4}$ but not after noncardiac surgery, ${ }^{5}$ suggesting that cardiovascular comorbidities indeed affect the response to COX-2 inhibition. Unfortunately, the swiftness with which COX-2 inhibitors were removed from the market has made more directed clinical evaluation of their safety difficult. 


$\begin{array}{|ll|}\text { Abbreviations and Acronyms } \\ \text { AAR } & =\text { area at risk } \\ \text { CX } & =\text { normal chow } \\ \text { COX } & =\text { cyclooxygenase } \\ \text { HCX } & =\text { high cholesterol diet } \\ \text { HDL } & =\text { high-density lipid (cholesterol) } \\ \text { LDL } & =\text { low-density lipid (cholesterol) } \\ \text { LV } & =\text { left ventricular } \\ \text { NV } & =\text { nonischemic left ventricular tissue } \\ \text { SDS- } & =\text { sodium dodecylsulfate- } \\ \text { PAGE } & \text { polyacrylamide gel electrophoresis } \\ \text { SOD } & =\text { superoxide dismutase }\end{array}$

Celecoxib is reported to have the most benign safety profile of all of the COX-2 inhibitors, ${ }^{6}$ perhaps related to its unique ability to improve endothelial function and smooth muscle relaxation in the setting of coronary artery disease. ${ }^{7}$ On the other hand, it has also been reported to increase vascular oxidative stress ${ }^{8}$ and to have antiangiogenic effects. ${ }^{9}$ We recently found that celecoxib improved blood flow in the ischemic myocardium of hypercholesterolemic pigs compared with untreated animals (Chu et al, submitted to Am J Physiol, 2011), an effect that was not seen in normocholesterolemic swine. ${ }^{9}$ Thus, cardiovascular comorbidities clearly influence the myocardial response to selective COX-2 inhibition with celecoxib. We designed this study to examine the effects of ischemia and hypercholesterolemia in the setting of celecoxib treatment.

\section{MATERIALS AND METHODS \\ Study Design}

Yorkshire miniswine (Parsons Research, Amherst, Mass) were fed either normal chow $(\mathrm{CX}, \mathrm{n}=6)$ or high cholesterol diet (Sinclair Research, Columbia, Mo) (HCX, $n=6)$. At 8 weeks of age, animals underwent placement of an Ameroid constrictor (Research Instruments SW, Escondido, Calif) on the left circumflex coronary artery. All animals were then started on oral celecoxib (200 mg once daily).

Seven weeks later, coronary angiography was performed. The heart was then exposed via midline sternotomy and physiologic measurements were taken, followed by blood draw and cardiac harvest. Nonischemic left ventricular (LV) tissue (NV) was harvested from directly adjacent to the left anterior descending coronary artery, while myocardium from the ischemic area at risk (AAR) was harvested from the left circumflex territory. Tissue samples were flash frozen in liquid nitrogen for subsequent analysis.

All experiments were approved by the Beth Israel Deaconess Medical Center and Rhode Island Hospital Institutional Animal Care and Use committees. Animals were cared for in accordance with the "Principles of Laboratory Animal Care" formulated by the National Society for Medical Research and the "Guide for the Care and Use of Laboratory Animals" (NIH publication No. 5377-3, 1996).

\section{Serum Lipid Profile}

Pig serum was analyzed for total cholesterol, high-density lipid (HDL) cholesterol, and triglycerides using a Beckman DXC 800 Chemistry Analyzer (Beckman Coulter Inc, Brea, Calif). Low-density lipid (LDL) cholesterol was calculated from these results using the formula: $\mathrm{LDL}=$ total cholesterol $-\mathrm{HDL}-($ triglycerides/5).

\section{Measurement of Global Myocardial Function}

Heart rate, mean arterial pressure, developed LV pressure, and LV contractility were recorded before cardiac harvest using single-sensor pressure catheters (Millar Instruments, Inc, Houston, Tex) introduced through the apex of the heart and into the abdominal aorta via a femoral artery sheath.

\section{Myocardial Perfusion Analysis}

Myocardial perfusion was measured via isotope-labeled microspheres (BioPAL, Worcester, Mass). Gold-labeled microspheres were injected during temporary occlusion of the left circumflex artery at the first procedure to demarcate the AAR. Microspheres labeled with lutetium (resting heart rate) and europium (pacing to 150 beats/min) were injected at the final procedure while blood was simultaneously withdrawn from the femoral artery catheter. Dried LV samples were exposed to neutron beams and microsphere densities measured using a gamma counter (BioPAL). Myocardial blood flow was determined using the following equation:

Blood flow $=($ withdrawal rate $/$ tissue weight $) \times$ (tissue microsphere count/blood microsphere count)

\section{Thrombogenic Ratio}

Tissue levels of the breakdown products of thromboxane $\mathrm{A}_{2}$ and prostacyclin, 11-dehydrothromboxane- $\mathrm{B}_{2}$ and 6-keto prostaglandin- $\mathrm{F}_{1 \alpha}$, respectively, were measured using enzyme-linked immunosorbent assay kits (Neogen Corp, Lexington, Ky). Tissue lysates underwent liquid-liquid exchange, extraction, and concentration, and were then loaded into 96-well plates containing antibody to either 11-dehydrothromboxane- $\mathrm{B}_{2}$ or 6-keto prostaglandin- $\mathrm{F}_{1 \alpha}$. After addition of colorimetric substrate, absorbance was read at $650 \mathrm{~nm}$. Results were expressed as a "thrombogenic ratio" or the ratio of 11-dehydrothromboxane- $\mathrm{B}_{2}$ to 6-keto prostaglandin- $\mathrm{F}_{1 \alpha}$.

\section{Immunoblotting}

Sixty micrograms of total protein from myocardial homogenates was fractionated by sodium dodecylsulfate-polyacrylamide gel electrophoresis (SDS-PAGE; Invitrogen, San Diego, Calif) and transferred to polyvinylidene difluoride membranes (Millipore, Bedford, Mass). Membranes were incubated with antibodies against prostacyclin synthase, thromboxane synthase (Cayman Chemical, Ann Arbor, Mich), myeloperoxidase (Sigma-Aldrich, St Louis, Mo), Cu/Zn-superoxide dismutase (Cu/Zn-SOD), and Mnsuperoxide dismutase (Mn-SOD; Enzo Life Sciences, Plymouth Meeting, $\mathrm{Pa}$ ) at dilutions recommended by the manufacturer, followed by the appropriate horseradish peroxidase-linked secondary antibodies (1:3000; Jackson ImmunoResearch, West Grove, Pa). Bands were visualized via chemiluminescence (Amersham, Piscataway, NJ) and photographed using GeneSnap software (Syngene, Cambridge, England). Densitometry was performed using Image J software (National Institutes of Health, Bethesda, Md). Ponceau staining was used to ensure equal protein loading.

Total protein oxidative stress was measured using the Oxyblot kit (Millipore, Billerica, Mass). Dinitrophenylhydrazine-derivatized tissue homogenates from the AAR were separated by SDS-PAGE and transferred to polyvinylidene difluoride membranes. Membranes were incubated with primary antibody to dinitrophenylhydrazine, followed by horseradish peroxidase-linked secondary antibody per manufacterer's recommendations. Immune complexes were visualized with chemiluminescence, and densitometry was applied to entire lanes to measure total protein oxidation.

\section{Immunohistochemical Staining for Capillary and Arteriolar Density}

Frozen myocardial sections, $12-\mu \mathrm{m}$ thick, were formalin fixed and then incubated with antibody against porcine CD31 (1:200; R\&D Systems, 

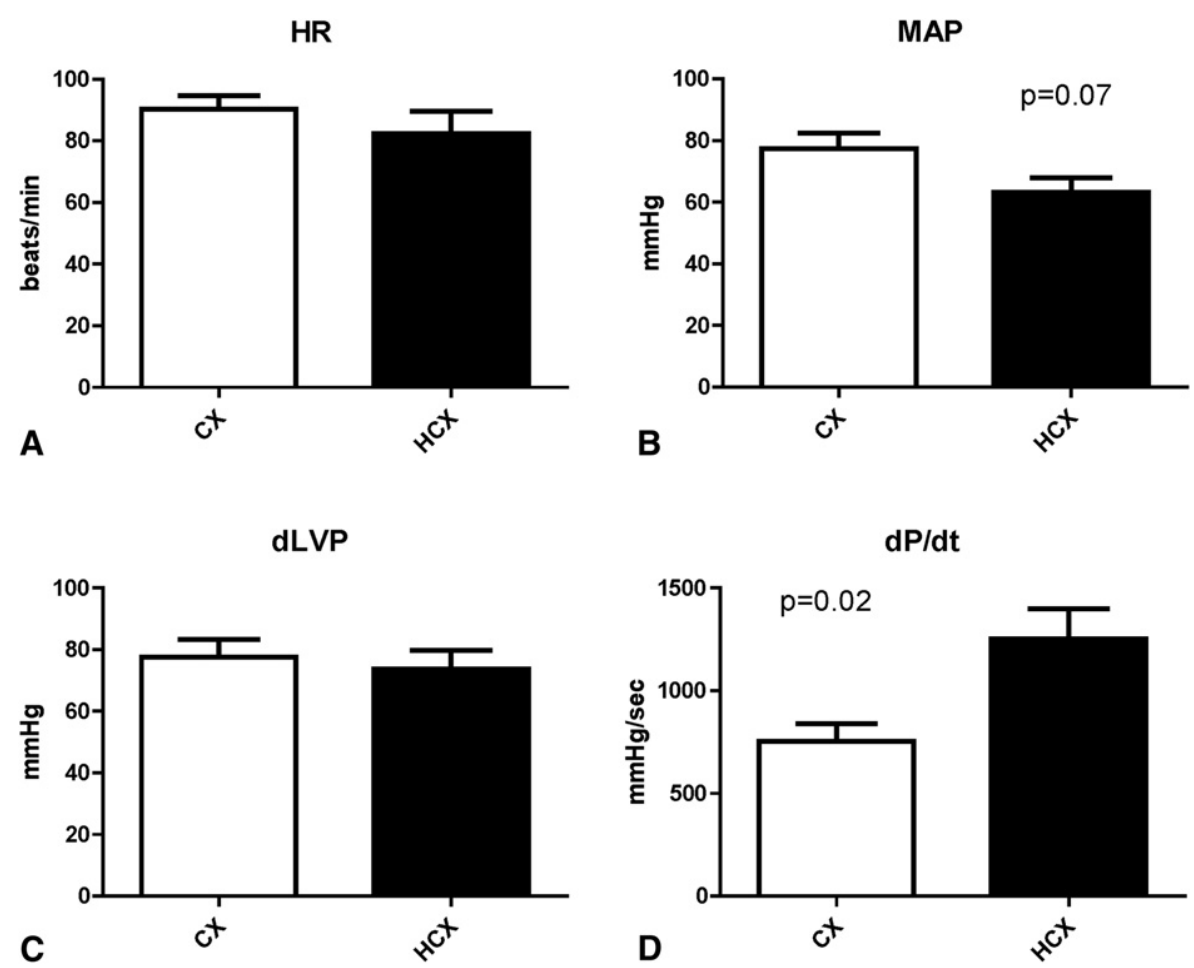

FIGURE 1. Hemodynamic parameters. Heart rate $(H R)$, mean arterial pressure $(M A P)$, developed left ventricular pressure $(d L V P)$, and LV contractility $(d P / d t)$ were measured at the time of cardiac harvest. There was no significant difference in HR (A), MAP (B), or dLVP (C) between groups, though MAP tended to be lower in the HCX group. The dLVP was significantly increased in the HCX group (D). CX, Normal chow; $H C X$, high cholesterol diet.

Minneapolis, Minn) and smooth muscle actin (1:400; Sigma-Aldrich), followed by approriate DyLight-conjugated secondary antibodies (1:100; Jackson ImmunoResearch, West Grove, Pa). Sections were mounted in Vectashield with 4',6-diamidino-2-phenylindole (Vector Laboratories, Burlingame, Calif). Three randomly selected photomicrographs were taken of each section with a Zeiss Axiolab microscope (Carl Zeiss, Inc, Thornwood, NY) at $20 \times$ magnification. Capillaries, defined as CD31-positive structures between 5 and $25 \mu \mathrm{m}^{2}$ in cross-sectional area, were counted using Image $\mathbf{J}$ software for each micrograph and averaged for each section. Arterioles were defined as structures that stained positively for both smooth muscle actin and CD31 and counted in a similar fashion. Data are presented as vessels per square millimeter.

\section{Statistical Analysis}

Results are presented as mean \pm standard error of the mean. Statistical comparisons were carried out with GraphPad Prism 5.0 Software (GraphPad Software Inc, San Diego, Calif) using a 2-way analysis of variance. The Newman-Keuls multiple comparison test was applied to test for significance between groups.

\section{RESULTS}

\section{Animal Model}

Of 7 animals originally included per group, 1 from each group died before cardiac harvest, presumably from cardiac arrhythmia, for a total 6 per group. All animals demonstrated complete occlusion of the left circumflex coronary artery at the time of cardiac harvest. Serum cholesterol values were significantly elevated in the HCX group
$(243.9 \pm 21.7 \mathrm{mg} / \mathrm{dL}$ vs $78.7 \pm 3.7 \mathrm{mg} / \mathrm{dL} ; P<.0001)$. HDL cholesterol was also increased in the HCX group $(60.5 \pm 8.4 \mathrm{mg} / \mathrm{dL}$ vs $27.8 \pm 2.32 \mathrm{mg} / \mathrm{dL} ; P<.01)$. However, the ratio of HDL/LDL cholesterol was significantly decreased in the HCX group compared with the CX group $(0.40 \pm 0.04 \mathrm{mg} / \mathrm{dL}$ vs $0.66 \pm 0.07 \mathrm{mg} / \mathrm{dL} ; P<.01)$.

LV contractility was significantly higher in the HCX group than in the CX group, but there was no difference in heart rate or LV pressure. There was a strong trend toward decreased mean arterial pressure in the HCX group, but this difference did not reach statistical significance (Figure 1, $A-D$ ).

Myocardial perfusion. Resting myocardial perfusion was significantly lower in the NV of hypercholesterolemic swine compared with normocholesterolemic swine and tended to be lower in the AAR as well. Overall, there was a significant effect of hypercholesterolemia in reducing myocardial perfusion at rest. Perfusion was similar between the AAR and the $\mathrm{NV}$ of both normal and hypercholesterolemic swine (Figure 2, A).

Similarly, there was a significant decrease in paced blood flow in the NV of hyperchoelsterolemic swine, but overall, hypercholesterolemia did not have a significant effect on paced blood flow (Figure 2, B).

Thrombogenic ratio. Thrombogenic ratio was significantly increased in the NV and AAR of hypercholesterolemic swine compared with normal diet swine. The 


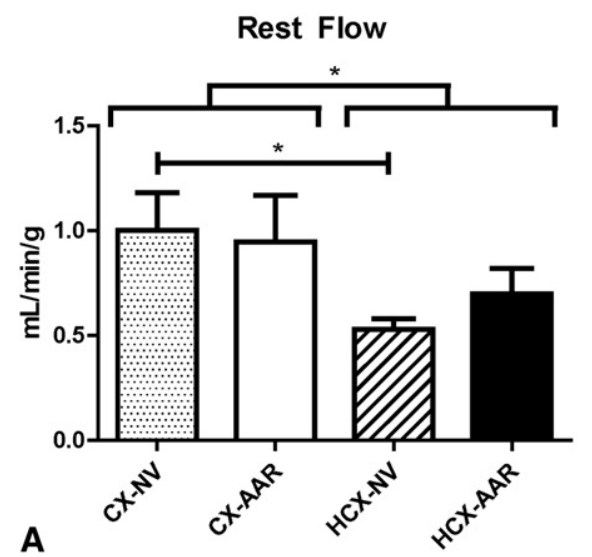

\section{Paced Flow}

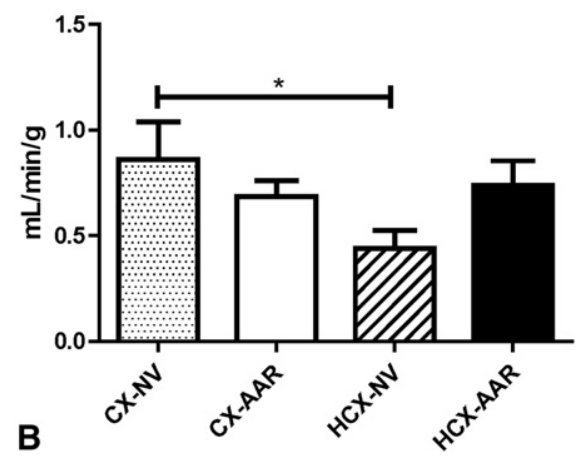

FIGURE 2. Myocardial blood flow. Blood flow in ischemic and nonischemic myocardium from both groups was measured by microsphere analysis. Under resting conditions (A), blood flow was significantly decreased in the NV of HCX swine compared with CX swine, and there was a significant overall effect of hypercholesterolemia in decreasing perfusion as well. Under ventricular pacing (B), blood flow was again decreased in the NV of HCX swine compared with CX swine, but there was no overall effect of hypercholesterolemic diet. $C X$, Normal chow; $H C X$, high cholesterol diet; $N V$, nonischemic left ventricular tissue; $A A R$, area at risk. $* P<.05$.

thrombogenic ratio was also lower in the AAR of normocholesterolemic animals compared with the NV. The same trend was seen in hypercholesterolemic animals, although this difference was not significant (Figure 3).

Protein expression. There was no difference in the expression of prostacyclin synthase or thromboxane synthase between groups or myocardial territories. Expression of myeloperoxidase, $\mathrm{Mn}-\mathrm{SOD}$, and $\mathrm{Cu} / \mathrm{Zn}-\mathrm{SOD}$ were increased in ischemic tissue compared with nonischemic

\section{Thrombogenic Ratio}

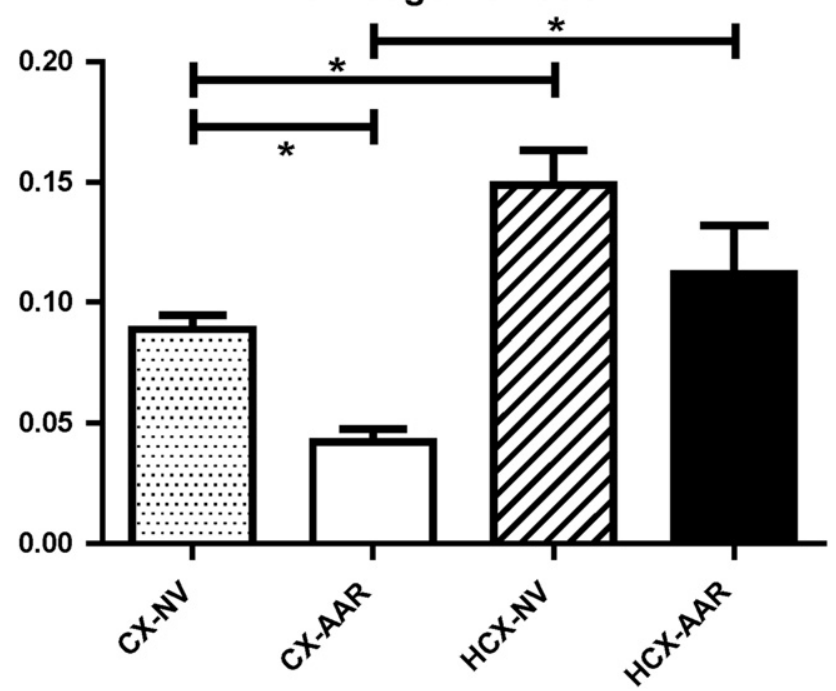

FIGURE 3. Thrombogenic ratio. Tissue levels of the stable metabolites of thromboxane and prostacyclin were measured and expressed as a ratio of 11-dehydrothromboxane- $\mathrm{B}_{2}$ to 6-keto-prostaglandin $\mathrm{F}_{1 \alpha}$. Thrombogenic ratio was increased in the HCX group, but it tended to be decreased in ischemic myocardium. $C X$, Normal chow; $H C X$, high cholesterol diet; $N V$, nonischemic left ventricular tissue; $A A R$, area at risk. $* P<.05$. tissues, but there was no diet-induced difference in expression (Figure 4, $A$ to $E$ ).

Protein oxidative stress. Total protein oxidative stress was increased in the ischemic territories of both groups, whereas hypercholesterolemia had no significant effect on protein oxidation (Figure $4, F$ ).

Vessel density. Neither capillary nor arteriolar density was significantly different between groups or myocardial territories, although arteriolar density did tend to be somewhat decreased in HCX animals (Figure 5).

\section{DISCUSSION}

Many previous studies have evaluated the effects of celecoxib on angiogenesis, thrombogenesis, oxidative stress, and inflammation, ${ }^{9-11}$ but this is the first to specifically study the effects of hypercholesterolemia and chronic ischemia, 2 conditions that are quite prevalent among the cardiac patient population, in the setting of celecoxib treatment.

The term "thrombogenic ratio" was first coined by Oubina and colleagues ${ }^{12}$ in 2001 . Several studies have since found that high oil intake and serum cholesterol, while increasing systemic levels of both thromboxane and prostacyclin, do not alter the thrombogenic ratio. ${ }^{12,13} \mathrm{~A}$ key finding in this study is that, in the setting of celecoxib treatment, hypercholesterolemia significantly increased the thrombogenic ratio in both ischemic and nonischemic myocardium, suggesting that hypercholesterolemia may affect prostanoid balance differently in the setting of celecoxib treatment. Although the expressions of thromboxane and prostacyclin synthases were not altered by a high-fat diet, hypercholesterolemia may shift thromboxane production in the heart to be regulated primarily by COX-1, such that selective inhibition of COX-2 preferentially inhibits prostacyclin production over thromboxane production. 

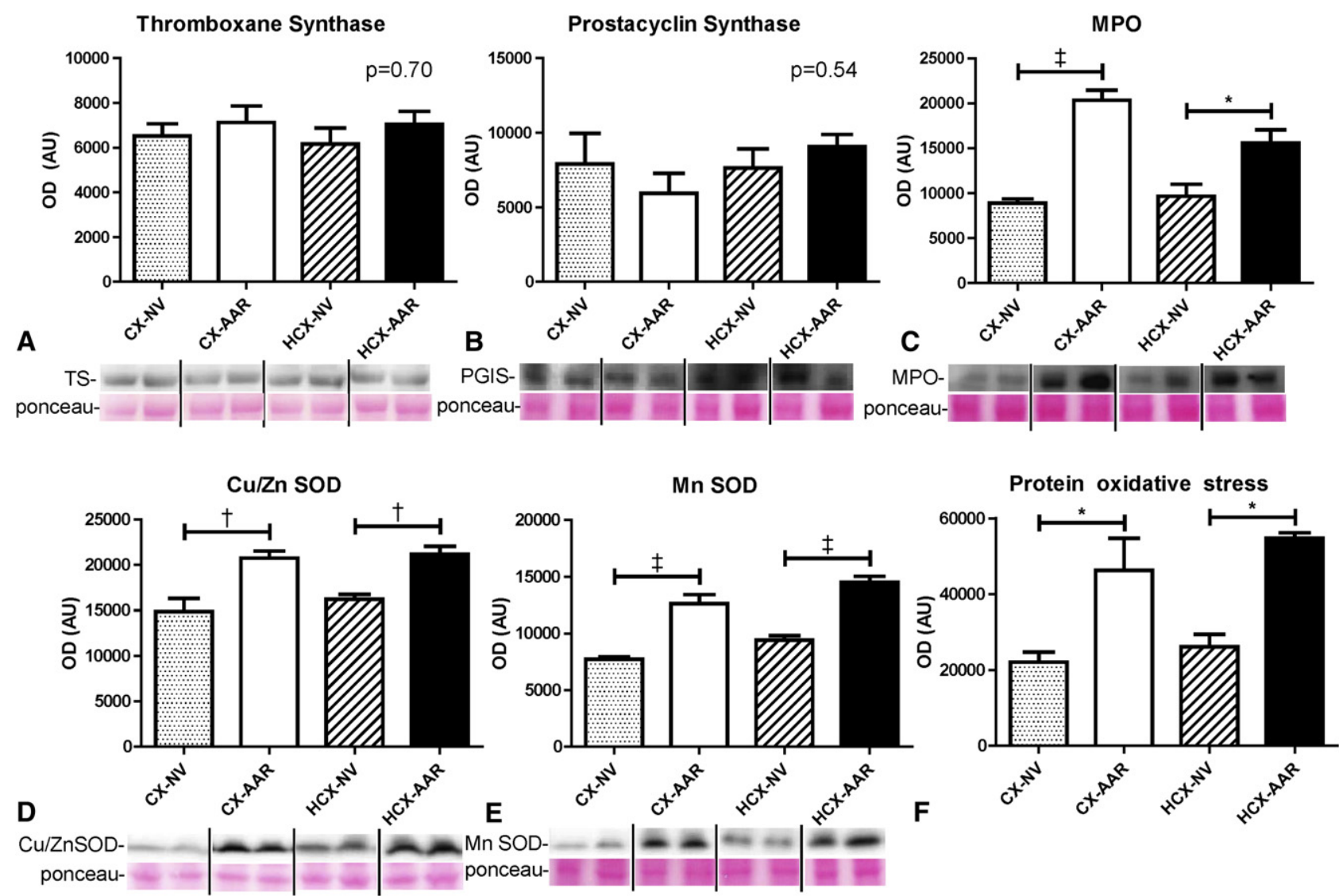

FIGURE 4. Immublotting results. Expression of thromboxane synthase, prostacyclin synthase, myeloperoxidase (MPO), Cu/Zn-superoxide dismutase $(\mathrm{Cu} / \mathrm{Zn}$-SOD), Mn-superoxide dismutase $(\mathrm{Mn}$-SOD), and total protein oxidative stress was measured in myocardial tissue lysates and expressed in arbitrary units of optical density $(O D)$. Shown are representative lanes from the same set of gels for each graph; images have been truncated for easier visualization. Expression of thromboxane synthase (A) and prostacyclin synthase (B) was not significantly different between groups, while MPO (C), Cu/ZN-SOD (D), Mn-SOD (E), and total protein oxidation (F) were all increased in ischemic myocardium compared with nonischemic myocardium. $* P<.05, \dagger P<.01$, $\ddagger P<.001$.

Although we did not specifically examine platelet aggregation in this study, a higher thrombogenic ratio could predispose to vasoconstriction and clot formation in the coronary vasculature of hypercholesterolemic patients taking celecoxib, leading to increased cardiovascular risk. Concordantly, we found that myocardial perfusion tended to be decreased in hypercholesterolemic animals in this study. The effect of HDL and LDL on prostanoid balance is quite controversial. Hypercholesterolemia has been shown to increase urinary excretion of thromboxane metabolites, ${ }^{14}$ and statin treatment effectively decreases urinary excretion of both thromboxane and prostacyclin metabolites ${ }^{15}$ but ours is the first study to specifically examine myocardial levels of thromboxane and prostacyclin. Yui and associates ${ }^{16}$ reported that HDL stabilizes circulating prostacyclin. It may be that although the HDL/LDL ratio was decreased in HCX animals, the increase in absolute HDL prolonged the action of myocardial prostacyclin on the coronary vasculature. Furthermore, prostanoid balance fluctuates in response to certain diseases that limit blood flow such as chronic myocardial ischemia, ${ }^{17}$ which may account for the decrease in thrombogenic ratio we saw in ischemic myocardium in this study. The interaction between cardiovascular comorbidities and prostanoid balance is obviously quite complex and requires further investigation.

Myocardial ischemia, hypercholesterolemia, and arachidonic acid metabolism are all associated with the release of reactive oxygen species. ${ }^{18}$ When sufficient reactive oxygen species are produced to overcome cellular defense mechanisms, they can damage or denature proteins, leading to enzyme deactivation, loss of contractile function, and vascular dysfunction. ${ }^{19}$ In this study, both total protein oxidative stress and myeloperoxidase, a sensitive marker for myocardial oxidation and inflammation, were increased in ischemic myocardium. Interestingly, although hypercholesterolemia has been shown in multiple models to increase oxidative stress, we did not observe this in celecoxibtreated swine. Celecoxib has been found to increase oxidative stress in healthy animals, ${ }^{20,21}$ but it actually decreases oxidative stress in models of malignancy ${ }^{22}$ and rats exposed 
Capillary density

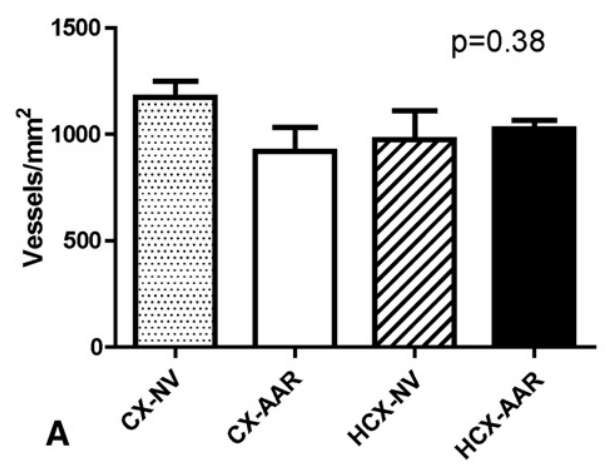

Arteriolar density

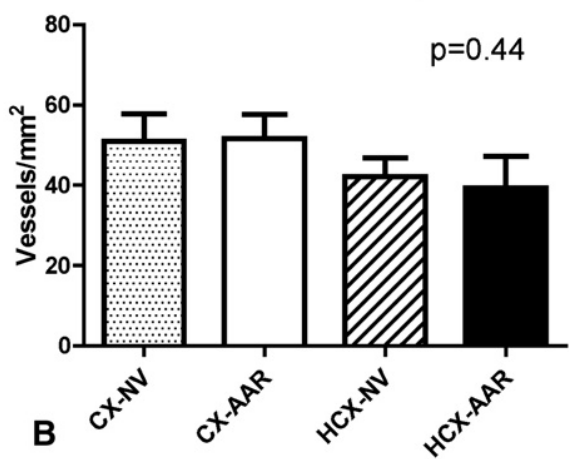

FIGURE 5. Vessel density. Capillary density and arteriolar density were measured by immunohistochemical staining for CD31 and smooth muscle actin, respectively, and expressed as vessels per square millimeter. There was no significant difference in capillary or vessel density between any of the groups. $C X$, Normal chow; $H C X$, high cholesterol diet; $N V$, nonischemic left ventricular tissue; $A A R$, area at risk.

to reactive oxygen species-rich cigarette smoke. ${ }^{23}$ Similarly, celecoxib may have decreased oxidative stress in the proinflammatory environment of chronic hypercholesterolemia in this study. We hypothesized that this antioxidant effect may have been mediated by increased expression of the freeradical scavenger SOD, but both $\mathrm{Cu} / \mathrm{Zn}-\mathrm{SOD}$ and $\mathrm{Mn}-\mathrm{SOD}$ were not upregulated in the hypercholesterolemic group. The increased SOD expression seen in the ischemic territories is likely a response to the increase in oxidative stress.

Overall, hypercholesterolemia produced minimal differences in ventricular function and angiogenesis as measured by vessel density in the present study. This was somewhat unexpected, inasmuch as previous studies by our group and others have shown that these parameters are negatively affected by hypercholesterolemia. ${ }^{24,25} \mathrm{LV}$ contractility was actually found to be increased in this study, and mean arterial pressure tended to be decreased in the HCX group. Recent evidence from our laboratory and others suggests that a beneficial effect of celecoxib may be responsible for this phenomenon. We recently reported that, specifically in hypercholesterolemic swine, celecoxib actually increases myocardial perfusion and function in the AAR (Chu et al, submitted to Am J Physiol, 2011), and others have shown that the drug acts as a peripheral vasodilator in patients with coronary artery disease. ${ }^{7}$ With so many potential clinical applications, celecoxib certainly warrants further investigation, but this study shows that cardiovascular comorbidities such as coronary artery disease and hypercholesterolemia must be taken into account when assessing the safety of celecoxib moving forward.

\section{LIMITATIONS}

This study has several potential limitations. First, the results of this study do not necessarily reflect what would be found in a clinical trial with thousands of patients. Certain relatively rare events may occur through nonvascular mechanisms that would only become evident in a large clinical trial. Second, COX inhibitors differ in their degree of selectivity for COX-1 and COX-2, so that the results of this study should not be generalized to other members of the class of drugs. Finally, inasmuch as the purpose of this study was to primarily assess the effects of hypercholesterolemia and chronic ischemia on the cardiovascular environment in the setting of celecoxib treatment, we did not include nondrug treated groups in this study. Thus, one should be careful in drawing conclusions about the specific cardiovascular effects of celecoxib from the data obtained in this study.

We thank the Animal Research Facility of Beth Israel Deaconess Medical Center and the Central Research Facility of Rhode Island Hospital for their excellent care of the animals used in this study.

\section{References}

1. Kujubu DA, Fletcher BS, Varnum BC, Lim RW, Herschman HR. TIS10, a phorbol ester tumor promoter-inducible mRNA from Swiss 3 T3 cells, encodes a novel prostaglandin synthase/cyclooxygenase homologue. J Biol Chem. 1991;266:12866-72.

2. Bombardier C, Laine L, Reicin A, Shapiro D, Burgos-Vargas R, Davis B, et al. Comparison of upper gastrointestinal toxicity of rofecoxib and naproxen in patients with rheumatoid arthritis. VIGOR Study Group. $N$ Engl J Med. 2000; 343:1520-8.

3. Nussmeier NA, Whelton AA, Brown MT, Langford RM, Hoeft A, Parlow JL, et al. Complications of the COX-2 inhibitors parecoxib and valdecoxib after cardiac surgery. N Engl J Med. 2005;352:1081-91.

4. Ott E, Nussmeier NA, Duke PC, Feneck RO, Alston RP, Snabes MC, et al. Efficacy and safety of the cyclooxygenase 2 inhibitors parecoxib and valdecoxib in patients undergoing coronary artery bypass surgery. $J$ Thorac Cardiovasc Surg. 2003; 125:1481-92.

5. Nussmeier NA, Whelton AA, Brown MT, Joshi GP, Langford RM, Singlai NK, et al. Safety and efficacy of the cyclooxygenase-2 inhibitors parecoxib and valdecoxib after noncardiac surgery. Anesthesiology. 2006;104:518-26.

6. McGettigan P, Henry D. Cardiovascular risk and inhibition of cyclooxygenase: a systematic review of the observational studies of selective and nonselective inhibitors of cyclooxygenase 2. JAMA. 2006;296:1633-44.

7. Chenevard R, Hurlimann D, Bechir M, Enseleit F, Spieker L, Hermann M, et al. Selective COX-2 inhibition improves endothelial function in coronary artery disease. Circulation. 2003;107:405-9.

8. Sodha NR, Clements RT, Feng J, Liu Y, Bianchi C, Horvath EM, et al. The effects of therapeutic sulfide on myocardial apoptosis in response to ischemia-reperfusion injury. Eur J Cardiothorac Surg. 2008;33:906-13. 
9. Robich MP, Chu LM, Feng J, Burgess TA, Laham RJ, Bianchi C, et al. Effects of selective cyclooxygenase-2 and nonselective cyclooxygenase inhibition on ischemic myocardium. J Thorac Cardiovasc Surg. 2010;140:1143-52.

10. Coon KD, Inge LJ, Swetel K, Felton V, Stafford P, Bremner RM. Genomic characterization of the inflammatory response initiated by surgical intervention and the effect of perioperative cyclooxygenase 2 blockade. $J$ Thorac Cardiovasc Surg. 2010;139:1253-60.

11. Gladding PA, Webster MW, Farrell HB, Zeng IS, Park R, Rujine N. The antiplatelet effect of six non-steroidal anti-inflammatory drugs and their pharmacodynamic interaction with aspirin in healthy volunteers. Am J Cardiol. 2008;101: 1060-3.

12. Oubina P, Sanchez-Muniz FJ, Rodenas S, Cuesta C. Eicosanoid production, thrombogenic ratio, and serum and LDL peroxides in normo- and hypercholesterolaemic post-menopausal women consuming two oleic acid-rich diets with different content of minor components. Br J Nutr. 2001;85:41-7.

13. Sanchez-Muniz FJ, Oubina P, Rodenas S, Benedi J, Cuesta C. Platelet aggregation, thromboxane production and thrombogenic ratio in postmenopausal women consuming high oleic acid-sunflower oil or palmolein. Eur J Nutr. 2003;42:299-306.

14. Davi G, Averna M, Catalano I, Barbagallo C, Ganci A, Notarbartolo A, et al. Increased thromboxane biosynthesis in type IIa hypercholesterolemia. Circulation. 1992;85:1792-8.

15. Puccetti L, Santilli F, Pasqui AL, Lattanzio S, Liani R, Ciani F, et al. Effects of atorvastatin and rosuvastatin on thromboxane-dependent platelet activation and oxidative stress in hypercholesterolemia. Atherosclerosis. 2011;214:122-8.

16. Yui Y, Aoyama T, Morishita H, Takahashi M, Takatsu Y, Kawai C. Serum prostacyclin stabilizing factor is identical to apolipoprotein A-1 (Apo A-1). A novel function of Apo A-1. J Clin Invest. 1988;82:803-7.

17. Sakai K, Ito K, Ogawa K. Roles of endogenous prostacyclin and thromboxane A2 in the ischemic canine heart. J Cardiovasc Pharmacol. 1982;4:129-35.

18. Oltman CL, Kane NL, Miller FJJ, Spector AA, Weintraub NL, Dellsperger KC. Reactive oxygen species mediate arachidonic acid-induced dilation in porcine coronary microvessels. Am J Physiol Heart Circ Physiol. 2003;285:H2309-15.

19. Misra MK, Sarwat M, Bhakuni P, Tuteja R, Tuteja N. Oxidative stress and ischemic myocardial syndromes. Med Sci Monit. 2009;15:RA209-19.

20. Sozer S, Diniz G, Lermioglu F. Effects of celecoxib in young rats: histopathological changes in tissues and alterations of oxidative stress/antioxidant defense system. Arch Pharm Res. 2011;34:253-9.

21. Robich MP, Chu LM, Burgess TA, Feng J, Bianchi C, Sellke FW. Effects of selective cyclooxygenase-2 and nonselective cyclooxygenase inhibition on myocardial function and perfusion. J Cardiovasc Pharmacol. 2011;57:122-30.

22. Kanwar SS, Vaiphei K, Nehru B, Sanyal SN. Antioxidative effects of nonsteroidal anti-inflammatory drugs during the initiation stages of experimental colon carcinogenesis in rats. J Environ Pathol Toxicol Oncol. 2008;27:89-100.

23. Koul A, Arora N. Celecoxib mitigates cigarette smoke induced oxidative stress in mice. Indian J Biochem Biophys. 2010;47:285-91.

24. Boodhwani M, Nakai Y, Mieno S, Voisine P, Bianchi C, Araujo EG, et al. Hypercholesterolemia impairs the myocardial angiogenic response in a swine model of chronic ischemia: role of endostatin and oxidative stress. Ann Thorac Surg. 2006; 81:634-41.

25. Rodriguez-Porcel M, Lerman A, Best PJ, Krier JD, Napoli C, Lerman LO. Hypercholesterolemia impairs myocardial perfusion and permeability: role of oxidative stress and endogenous scavenging activity. J Am Coll Cardiol. 2001;37:608-15.

\section{Discussion}

Dr Michael E. Jessen (Dallas, Tex). I enjoyed your study, but several of the observation that you made seemed counterintuitive.
For example, what is your explanation for why what you call contractility actually was greater in the ischemic zones?

Dr Chu. That is a very good question. We were surprised by that as well. I think the other surprising thing that we also found was that mean arterial pressure was actually decreased in the high-cholesterol animals. The improvement in contractility may have some relationship with the decreased mean arterial pressure and decreased afterload, but we are not really sure why that is. It may be an effect of the drug celecoxib (Celebrex), but without having a controlled, non-drug-treated group, it would be hard to make that conclusion.

Dr Jessen. If what you are calling contractility is affected by afterload, then it is really not contractility by a pure definition. Did you consider doing anything to measure dimensions in those 2 zones to try to get a better handle on that?

Dr Chu. Unfortunately, we did not; we did not do any echo or any pressure-volume loop analysis. We are moving into that realm now, so I hope in future studies we will be able to look at those things.

Dr Jessen. The other thing was that the contractility was increased, but this was in areas where there was decreased blood flow. Was that what I took from your talk?

Dr Chu. There was a trend toward decreased blood flow, but it was not statistically significant.

Dr Jessen. That would be an unusual combination of events as well. Finally, what is your explanation for the ischemic areas seeming to have a decreased thrombogenic ratio? That seems counterintuitive as well.

Dr Chu. That is a great question as well. As you know, thromboxane and prostacyclin are partially released by the endothelium in tissues in the body. It is possible that ischemia affects the balance of the release of those prostanoids to favor prostacyclin release, thus decreasing the thrombogenic ratio. Again, the explanation for that is uncertain at this point.

Dr Patrick M. McCarthy (Chicago, Ill). The bottom line question is, if your dad was receiving celecoxib and that was the last available drug that actually works well for his arthritis, how would you balance these factors?

Dr Chu. Right. That is the million dollar question. However, this study was not really adequately designed to specifically assess the safety profile of celecoxib because we did not include a nondrug-treated group. However, we have done another study that is currently under review comparing celecoxib use in high-cholesterol pigs to non-drug-treated high-cholesterol pigs. It does surprise me to have some beneficial effects with regard to reducing oxidative stress, actually increasing blood flow in the ischemic territory. I think definitely the safety profile of the drug deserves further investigation. 